\title{
On a New Bimodal Normal Family
}

\author{
Sayed Mohammad Reza Alavi \\ Shahid Chamran University
}

\begin{abstract}
The unimodal distributions are frequently used in the theorical statistical studies. But in applied statistics, there are many situations in which the unimodal distributions can not be fitted to the data. For example, the distribution of the data outside the control zone in quality control or outlier observations in linear models and time series may required to be a bimodal. These situations, occur when the recorded data have the probability proportional to an increasing function of absolute value of deviations. In this paper a new family of distributions called double normal family of distribution is introduced and characterized. This symmetric family is a subclass of the univariate Kotz type distributions. The normal distribution is a special case of this family. Estimation of location and scale parameters by moment and maximum likelihood methods are given. Some pivotal quantity are introduced. Confidence intervals for some parameters by numerical methods are given.
\end{abstract}

Keywords. double normal family; standard double normal; confidence interval; point estimation; pivotal quantity.

MSC 2010: 60E05, 62E10.

\section{Introduction}

The unimodal distributions are frequently used in the theorical statistical studies. But in applied statistics, there are many situations in which the unimodal distributions can not be fitted to the data. For example, the distribution of the data outside the control zone in quality control or outlier observations in linear models and time series may required to be a bimodal. 
These situations, occur when the recorded data have the probability proportional to an increasing function of absolute value of deviations. In this paper a new family of distributions is introduced as a generalized normal distribution. This family which is a subclass of the univariate Kotz type distributions (see Fang and et al., 1999; and Nadarajah, 2003 ) is called by double normal family and denoted by $D N(\mu, \sigma, \beta)$ with the following probability density function (pdf)

$$
f(x ; \mu, \sigma, \beta)=\frac{|x-\mu|^{\beta}}{\Gamma\left(\frac{\beta+1}{2}\right) 2^{\frac{\beta+1}{2}} \sigma^{\beta+1}} \exp \left\{\frac{-1}{2 \sigma^{2}}(x-\mu)^{2}\right\} \quad-\infty<x<\infty,
$$

where $-\infty<\mu<\infty, \quad \sigma>0$ and $\beta \geqslant 0$.

The $\mu, \sigma$ and $\beta$ are location, scale and shape parameters, respectively. When $\beta=0$, this distribution reduces to the normal distribution. When $\beta>0$, this family has two symmetric mode about $\mu$. This family can be used for the ballistic data which the observations with more far from the target are interested to researchers. This family can be considered as a weighted normal distribution with weight function $w(x)=|x-\mu|^{\beta}$. The weighed distributions have been studied comprehensively by Patil (2002) and Patil and Rao (1977), (see also Alavi and Chinipardaz, 2009). The double normal distribution discussed by Alavi and Chinipardaz (2011) is a special case of this family when $\beta=2$. The outline of this paper is as follows. In Section 2 common properties of $D N(\mu, \sigma, \beta)$ are studied. The point estimations and confidence intervals are discussed in Sections 3 and 4 . The confidence interval of parameters using numerical study is given in Sections 5 and 6 .

\section{Common Properties of Double Normal Family}

In this section some properties of double normal family are studied. Suppose that $X \sim D N(\mu, \sigma, \beta)$, pdf's of $X$ for $\mu=0, \sigma=1$ and $\beta=0,1,2$ are shown in Figure 1. From this figure, One can realize that this family is symmetric.

The distributions are all bimodal except for $\beta=0$. When $\mu=0$ and $\sigma=$ 1 , we call it as standard double normal of order $\beta$ and denote it by $S D N(\beta)$. The mean, variance and cdf are obtained from the following theorem.

Theorem 1. Suppose that $X \sim D N(\mu, \sigma, \beta)$. Then 


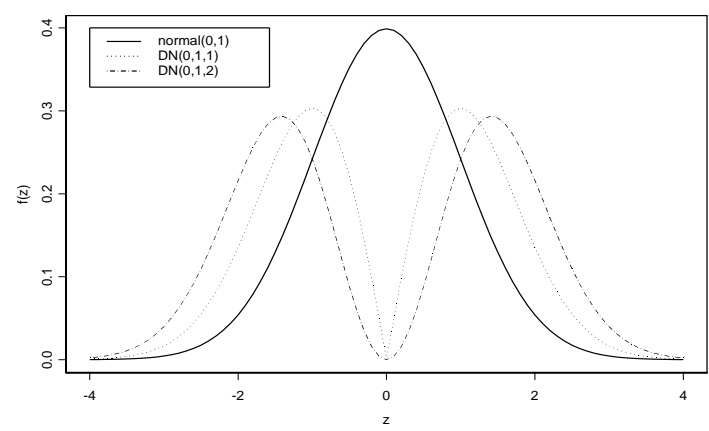

Figure 1. pdf's of standard normal and standard double normalwith $\beta=1$ and $\beta=2$

a) The mean and variance of $X$ are $\mu$ and $(\beta+1) \sigma^{2}$, respectively.

b) The cumulative distribution function of $X$ is given by

$$
F_{X}(x)= \begin{cases}\frac{1}{2}-\frac{1}{2} F_{T}\left(\frac{(x-\mu)^{2}}{\sigma^{2}}\right) & x<\mu \\ \frac{1}{2}+\frac{1}{2} F_{T}\left(\frac{(x-\mu)^{2}}{\sigma^{2}}\right) & x \geqslant \mu\end{cases}
$$

where $F_{T}(\cdot)$ is cdf of random variable $T$ distributed as gamma distribution $\left(\frac{\beta+1}{2}, \frac{1}{2}\right)$ denoted by $\operatorname{Gamma}\left(\frac{\beta+1}{2}, \frac{1}{2}\right)$.

Proof. (a) It is sufficient to show that $E(X-\mu)=0$. We have

$$
\begin{aligned}
E(X-\mu) & =\int_{-\infty}^{\infty}(x-\mu) \frac{|x-\mu|^{\beta}}{\Gamma\left(\frac{\beta+1}{2}\right) 2^{\frac{\beta+1}{2}} \sigma^{\beta+1}} \exp \left\{\frac{-1}{2 \sigma^{2}}(x-\mu)^{2}\right\} d x \\
& =\int_{-\infty}^{\infty} z \frac{|z|^{\beta}}{\Gamma\left(\frac{\beta+1}{2}\right) 2^{\frac{\beta+1}{2}} \sigma^{\beta+1}} \exp \left\{\frac{-1}{2 \sigma^{2}}(z)^{2}\right\} d z
\end{aligned}
$$

where $z=\frac{x-\mu}{\sigma}$. Since, the intergrand is an odd function in term of $z$, the 
intergral becomes zero. Similarly, for the variance term, we have

$$
\begin{aligned}
E(X-\mu)^{2} & =\int_{-\infty}^{\infty}(x-\mu)^{2} \frac{|x-\mu|^{\beta}}{\Gamma\left(\frac{\beta+1}{2}\right) 2^{\frac{\beta+1}{2}} \sigma^{\beta+1}} \exp \left\{\frac{-1}{2 \sigma^{2}}(x-\mu)^{2}\right\} d x \\
& =\int_{-\infty}^{\infty} \frac{|z|^{\beta+2}}{\Gamma\left(\frac{\beta+1}{2}\right) 2^{\frac{\beta+1}{2}} \sigma^{\beta+1}} \exp \left\{\frac{-1}{2 \sigma^{2}} z^{2}\right\} d z \\
& =2 \int_{0}^{\infty} \frac{z^{\beta+2}}{\Gamma\left(\frac{\beta+1}{2}\right) 2^{\frac{\beta+1}{2}} \sigma^{\beta+1}} \exp \left\{\frac{-1}{2 \sigma^{2}} z^{2}\right\} d z .
\end{aligned}
$$

Using the transformation $Y=\frac{Z^{2}}{\sigma^{2}}$ and after some algebra calculations, $E(X-$ $\mu)^{2}=(\beta+1) \sigma^{2}$.

(b) We set

$$
\begin{aligned}
F_{X}(u)= & \int_{-\infty}^{u} \frac{|x-\mu|^{\beta}}{\Gamma\left(\frac{\beta+1}{2}\right) 2^{\frac{\beta+1}{2}} \sigma^{\beta+1}} \exp \left\{-\frac{1}{2 \sigma^{2}}(x-\mu)^{2}\right\} d x \\
& = \begin{cases}\int_{-\infty}^{\frac{u-\mu}{\sigma}} \frac{|z|^{\beta}}{\Gamma\left(\frac{\beta+1}{2}\right) 2^{\frac{\beta+1}{2}}} \exp \left\{-\frac{1}{2} z^{2}\right\} d z & u<\mu \\
\frac{1}{2}+\int_{0}^{\frac{u-\mu}{\sigma}} \frac{|z|^{\beta}}{\Gamma\left(\frac{\beta+1}{2}\right) 2^{\frac{\beta+1}{2}}} \exp \left\{-\frac{1}{2} z^{2}\right\} d z & u \geqslant \mu\end{cases} \\
& = \begin{cases}\frac{1}{2} \int_{\left(\frac{u-\mu}{\sigma}\right)^{2}}^{\infty} \frac{y^{\frac{\beta-1}{2}}}{\Gamma\left(\frac{\beta+1}{2}\right) 2^{\frac{\beta+1}{2}}} \exp \left\{-\frac{1}{2} y\right\} d y & u<\mu \\
\frac{1}{2}+\frac{1}{2} \int_{0}^{\left(\frac{u-\mu}{\sigma}\right)^{2}} \frac{y^{\frac{\beta-1}{2}}}{\Gamma\left(\frac{\beta+1}{2}\right) 2^{\frac{\beta+1}{2}}} \exp \left\{-\frac{1}{2} y\right\} d y & u \geqslant \mu .\end{cases}
\end{aligned}
$$

Thus, the proof is completed.

The following theorem explains that any liner combination of double normal distribution is also a double normal.

Theorem 2. Suppose that $X \sim D N(\mu, \sigma, \beta)$, then for $a \neq 0, a X+b \sim$ $D N(a \mu+b,|a| \sigma, \beta), a, b \in R$.

Proof. Trivial. 
Theorem 3. Suppose that $X \sim D N(\mu, \sigma, \beta)$. Let $Z=\frac{X-\mu}{\sigma}$, then

a) $Z \sim S D N(\beta)$.

b) $Z^{2} \sim \operatorname{Gamma}\left(\frac{\beta+1}{2}, \frac{1}{2}\right)$.

c) $|Z| \sim \operatorname{HSDN}(\beta)$, where $H S D N(\beta)$ denoted Half-Double Normal distribution with pdf given by

$$
f_{Y}(y)=\frac{2 y^{\beta}}{2^{\frac{\beta+1}{2}} \Gamma\left(\frac{\beta+1}{2}\right)} \exp \left\{-\frac{1}{2} y^{2}\right\}
$$

Proof. (a) The proof is resulted from Theorem 2.

(b) Using the transformation $Y=Z^{2}$, then the cumulative distribution function (cdf) of $\mathrm{Y}$ is given by

$$
F_{Y}(y)=2 F_{Z}(\sqrt{y})
$$

where $F_{Z}(\cdot)$ is cdf of $S D N(\beta)$. Thus pdf of $Y$ is given by

$$
f_{Y}(y)=\frac{f_{Z}(\sqrt{y})}{\sqrt{y}}=\frac{1}{2^{\frac{\beta+1}{2}} \Gamma\left(\frac{\beta+1}{2}\right)} y^{\frac{\beta-1}{2}} \exp \left\{-\frac{1}{2} y\right\}
$$

which is a $\operatorname{Gamma}\left(\frac{\beta+1}{2}, \frac{1}{2}\right)$.

(c) It is trivial.

The moment generating function (mgf) of $S D N(\beta)$ is given in the following theorem.

Theorem 4. Suppose that $Z \sim S D N(\beta)$. Then

a) $m g f$ of $Z, M_{Z}(t)$, is

$$
M_{Z}(t)=\frac{\sqrt{2 \pi}}{2^{\frac{\beta+1}{2}} \Gamma\left(\frac{\beta+1}{2}\right)} \exp \left\{\frac{1}{2} t^{2}\right\} E\left|Z^{*}\right|^{\beta}
$$

where $Z^{*} \sim N(t, 1)$.

b) The q-th quantile of $Z$ is

$$
F_{Z}^{-1}(q)= \begin{cases}-\sqrt{F_{T}^{-1}(1-2 q)} & q<0.5 \\ \sqrt{F_{T}^{-1}(2 q-1)} & q \geqslant 0.5\end{cases}
$$


Proof. (a) We have

$$
\begin{aligned}
M_{Z}(t) & =\int_{-\infty}^{\infty} \frac{1}{2^{\frac{\beta+1}{2}} \Gamma\left(\frac{\beta+1}{2}\right)} \exp \{t z\}|z|^{\beta} \exp \left\{-\frac{1}{2} z^{2}\right\} d z \\
& =\frac{\sqrt{2 \pi}}{2^{\frac{\beta+1}{2}} \Gamma\left(\frac{\beta+1}{2}\right)} \exp \left\{\frac{1}{2} t^{2}\right\} \int_{-\infty}^{\infty} \frac{|z|^{\beta}}{\sqrt{2 \pi}} \exp \left\{-\frac{1}{2}(z-t)^{2}\right\} d z \\
& =\frac{\sqrt{2 \pi}}{2^{\frac{\beta+1}{2}} \Gamma\left(\frac{\beta+1}{2}\right)} \exp \left\{\frac{1}{2} t^{2}\right\} E\left|Z^{*}\right|^{\beta} .
\end{aligned}
$$

(b) The proof is resulted from Theorem 1 .

For example, the mgf of $S D N(1)$ is

$$
M_{Z}(t)=1+\frac{\sqrt{2 \pi}}{2} t \exp \left\{\frac{1}{2} t^{2}\right\}\{1-2 \Phi(-t)\}
$$

where $\Phi(\cdot)$ is cdf of standard normal distribution. As an another example, the mgf of $S D N(2)$ is given by

$$
M_{Z}(t)=\left(1+t^{2}\right) \exp \left\{\frac{1}{2} t^{2}\right\} .
$$

The pdf, cdf, and quantiles of $S D N(\beta)$ are calculated numerically using SPLUS-2000 software as the programmig tool. We also generate a sample from $S D N(\beta)$ from the above written codes. In the next section, the point estimation of parameters will be illustrated.

\section{Point Estimation of Parameters}

For the specified $\beta$, the moment estimator of $\mu$ and $\sigma^{2}$ are $\bar{X}=\frac{\sum_{i=1}^{n} X_{i}}{n}$ and $\frac{\sum_{i=1}^{n}\left(X_{i}-\bar{X}\right)^{2}}{n(\beta+1)}$, respectively. When $\beta$ is unknown the moment estimator of $\mu$ is $\bar{X}=\frac{\sum_{i=1}^{n} X_{i}}{n}$ but the moment estimator of $\sigma^{2}$ and $\beta$ are very complicated. The maximum likelihood estimator (mle) of $\sigma^{2}$ when $\beta$ and $\mu$ are known is $\frac{\sum_{i=1}^{n}\left(X_{i}-\mu\right)^{2}}{n(\beta+1)}$, for other cases mle of parameters are obtained from the following equations.

$$
\beta \sum_{i=1}^{n} \frac{1}{\left(x_{i}-\mu\right)}=\frac{1}{\sigma^{2}} \sum_{i=1}^{n}\left(x_{i}-\mu\right)
$$




$$
\begin{gathered}
\sigma^{2}=\frac{\sum_{i=1}^{n}\left(x_{i}-\mu\right)^{2}}{n(\beta+1)} \\
\sum_{i=1}^{n} \log \left|x_{i}-\mu\right|=n \frac{\partial}{\partial \beta} \log \nu
\end{gathered}
$$

where $\nu=\Gamma\left(\frac{\beta+1}{2}\right) 2^{\frac{\beta+1}{2}} \sigma^{\beta+1}$. Such equations are usually solved by NewtonRaphson iterative method

$$
\hat{\boldsymbol{\theta}}^{(k+1)}=\hat{\boldsymbol{\theta}}^{(k)}-\left[\frac{\partial^{2} \log L}{\partial \theta_{i} \partial \theta_{j}}\right]_{\hat{\boldsymbol{\theta}}^{(k)}}^{-1}\left[\frac{\partial \log L}{\partial \boldsymbol{\theta}}\right]_{\hat{\boldsymbol{\theta}}^{(k)}},
$$

where $L$ is likelihood function and $\hat{\boldsymbol{\theta}}^{(j)}=\left(\hat{\mu}^{(j)}, \hat{\sigma}^{(j)}, \hat{\beta}^{(j)}\right)^{\prime}$ is the $j$ th step of estimation of $(\mu, \sigma, \beta)^{\prime}$. The procedure continue to $\left|\hat{\theta}_{i}^{(k+1)}-\hat{\theta}_{i}^{(k)}\right|<\varepsilon_{i}$, $i=1,2,3$, where $\varepsilon_{i}$ is an arbitrary small value chosen by the researcher. Thus we have

$$
\left[\frac{\partial \log L}{\partial \boldsymbol{\theta}}\right]_{\hat{\boldsymbol{\theta}}^{(k)}}=\left(T_{1}, T_{2}, T_{3}\right)^{\prime}
$$

and

$$
\left[\frac{\partial^{2} \log L}{\partial \theta_{i} \partial \theta_{j}}\right]_{\hat{\boldsymbol{\theta}}^{(k)}}=\left(\begin{array}{ccc}
A & -2 \sum \frac{\left(x_{i}-\hat{\mu}^{(k)}\right)}{\hat{\sigma}^{3(k)}} & D \\
-2 \sum \frac{\left(x_{i}-\hat{\mu}^{(k)}\right)}{\hat{\sigma}^{3(k)}} & B & -\frac{4 n}{\hat{\sigma}^{(k)}} \\
D & -\frac{4 n}{\hat{\sigma}^{(k)}} & C
\end{array}\right)
$$

where

$$
\begin{gathered}
T_{1}=-\hat{\beta}^{(k)} \sum \frac{1}{\left(x_{i}-\hat{\mu}^{(k)}\right)}+\frac{\sum\left(x_{i}-\hat{\mu}^{(k)}\right)}{\hat{\sigma}^{2(k)}}, \\
T_{2}=-\frac{n\left(\hat{\beta}^{(k)}+1\right)}{\hat{\sigma}^{(k)}}+\frac{\sum\left(x_{i}-\hat{\mu}^{(k)}\right)^{2}}{\hat{\sigma}^{3(k)}}, \\
T_{3}=\sum \log \left(x_{i}-\hat{\mu}^{(k)}\right)^{2}-\frac{n \Gamma^{\prime}\left(\frac{\hat{\beta}^{(k)}+1}{2}\right)}{\Gamma\left(\frac{\hat{\beta}^{(k)}+1}{2}\right)}-n \log 2-4 n \log \hat{\sigma}^{(k)}, \\
A=-\hat{\beta}^{(k)} \sum \frac{1}{\left(x_{i}-\hat{\mu}^{(k)}\right)^{2}}-\frac{n}{\hat{\sigma}^{2(k)}}, \\
B=\frac{n\left(\hat{\beta}^{(k)}+1\right)}{\hat{\sigma}^{2(k)}}-3 \sum_{i=1}^{n} \frac{\left(x_{i}-\hat{\mu}^{(k)}\right)^{2}}{\hat{\sigma}^{4(k)}},
\end{gathered}
$$




$$
C=-\frac{n \Gamma^{\prime \prime}\left(\frac{\hat{\beta}^{(k)}+1}{2}\right) \Gamma\left(\frac{\hat{\beta}^{(k)}+1}{2}\right)}{\left(\Gamma\left(\frac{\hat{\beta}^{(k)}+1}{2}\right)\right)^{2}}
$$

and

$$
D=-2 \sum \frac{1}{\left(x_{i}-\hat{\mu}^{(k)}\right)} .
$$

The scoring method (Knight, 2000; and Stuart et al., 1999) is an alternative method. However, they provide better results, but, its convergence rate is slower. Based on this method $\frac{\partial^{2} \log L}{\partial \theta_{i} \partial \theta_{j}}$ is replaced with $E\left(\frac{\partial^{2} \log L}{\partial \theta_{i} \partial \theta_{j}}\right)=$ $-(\operatorname{var}(\hat{\boldsymbol{\theta}}))^{-1}$. Furthermore

$$
\hat{\boldsymbol{\theta}}^{(k+1)}=\hat{\boldsymbol{\theta}}^{(k)}-[\operatorname{var}(\hat{\boldsymbol{\theta}})]_{\hat{\boldsymbol{\theta}}^{(k)}}\left[\frac{\partial \log L}{\partial \boldsymbol{\theta}}\right]_{\hat{\boldsymbol{\theta}}^{(k)}} .
$$

where $\left[\frac{\partial \log L}{\partial \boldsymbol{\theta}}\right]_{\hat{\boldsymbol{\theta}}^{(k)}}$ is given in (5) and

$$
[\operatorname{var}(\hat{\boldsymbol{\theta}})]_{\hat{\boldsymbol{\theta}}^{(k)}}^{-1}=\left(\begin{array}{ccc}
\frac{n\left(2 \hat{\beta}^{(k)}-1\right)}{\left(\hat{\beta}^{(k)}-1\right) \hat{\sigma}^{2(k)}} & 0 & 0 \\
0 & \frac{n\left(2 \hat{\beta}^{(k)}-7\right)}{\hat{\sigma}^{2(k)}} & \left.\frac{4 n}{\frac{\hat{\sigma}^{(k)}}{2}}\right) \Gamma\left(\frac{\hat{\beta}^{(k)}+1}{2}\right) \\
0 & \frac{4 n}{\hat{\sigma}^{(k)}} & \frac{n \Gamma^{\prime \prime}\left(\frac{\hat{\beta}^{(k)}}{2}\right.}{\left(\Gamma\left(\frac{\hat{\beta}^{(k)}+1}{2}\right)\right)^{2}}
\end{array}\right) .
$$

These procedures need the initial values, $\hat{\boldsymbol{\theta}}^{(0)}=\left(\hat{\mu}^{(0)}, \hat{\sigma}^{(0)}, \hat{\beta}^{(0)}\right)^{\prime}$. One approprate suggestion can be estimators $\mu$ and $\sigma^{2}$ based on the moment method with an initial value for $\hat{\beta}^{(0)}$. As the result, $\hat{\boldsymbol{\theta}}^{(0)}=\left(\bar{x}, \sqrt{\frac{\sum\left(x_{i}-\bar{x}\right)^{2}}{n\left(\hat{\beta}^{(0)}+1\right)}}, \hat{\beta}^{(0)}\right)^{\prime}$. The asymptotic distribution based on the maximum likelihood estimators is

$$
\left(\begin{array}{c}
\hat{\mu} \\
\hat{\sigma} \\
\hat{\beta}
\end{array}\right) \sim N_{3}\left(\left(\begin{array}{c}
\mu \\
\sigma \\
\beta
\end{array}\right), \Sigma\right)
$$

where

$$
\boldsymbol{\Sigma}^{-1}=\left(\begin{array}{ccc}
\frac{n(2 \beta-1)}{(\beta-1) \sigma^{2}} & 0 & 0 \\
0 & \frac{n(2 \beta-7)}{\sigma^{2}} & \frac{4 n}{\sigma} \\
0 & \frac{4 n}{\sigma} & \frac{n \Gamma^{\prime \prime}\left(\frac{\beta+1}{2}\right) \Gamma\left(\frac{\beta+1}{2}\right)}{\left(\Gamma\left(\frac{\beta+1}{2}\right)\right)^{2}}
\end{array}\right)
$$


However, for known $\beta>1$, which applied in the weighted sampling form normal distribution, one important problem for the mle's is their asymptotically properties. For large $n$ we have

$$
\left(\begin{array}{l}
\hat{\mu} \\
\hat{\sigma}
\end{array}\right) \sim N_{2}\left(\left(\begin{array}{l}
\mu \\
\sigma
\end{array}\right),\left(\begin{array}{cc}
\frac{(\beta-1) \sigma^{2}}{n(2 \beta-1)} & 0 \\
0 & \frac{\sigma^{2}}{2 n(\beta+1)}
\end{array}\right)\right) .
$$

For example, if $\beta=2$ (see Alavi and Chinipardaz, 2011)

$$
\left(\begin{array}{c}
\hat{\mu} \\
\hat{\sigma}
\end{array}\right) \sim N_{2}\left(\left(\begin{array}{l}
\mu \\
\sigma
\end{array}\right),\left(\begin{array}{cc}
\frac{\sigma^{2}}{3 n} & 0 \\
0 & \frac{\sigma^{2}}{6 n}
\end{array}\right)\right) .
$$

In the next section, the exact and asympototic confidence intervals for $\mu$ and $\sigma$ are derived when $\beta$ is known.

\section{Confidence Interval of Parameters}

Suppose that $X_{1}, X_{2}, \ldots, X_{n}$ is a random sample from $D N(\mu, \sigma, \beta)$. When $\mu$ is known, one explicit CI can be drawn for $\sigma$ using the following theorem.

Theorem 5. Suppose $X_{1}, X_{2}, \ldots, X_{n}$ be a random sample from $D N(\mu, \sigma, \beta)$. Then

$$
\frac{\sum\left(X_{i}-\mu\right)^{2}}{\sigma^{2}} \sim \operatorname{Gamma}\left(n\left(\frac{\beta+1}{2}\right), \frac{1}{2}\right)
$$

Proof. Proof can be constructed from Theorem 3 .

The pivotal quantity in (11) leads to the following CI for $\sigma$

$$
\left(\frac{\sqrt{(\beta+1) n \hat{\sigma}}}{\sqrt{G\left(n\left(\frac{\beta+1}{2}\right), \frac{1}{2}, 1-\frac{\alpha}{2}\right)}}, \frac{\sqrt{(\beta+1) n \hat{\sigma}}}{\sqrt{G\left(n\left(\frac{\beta+1}{2}\right), \frac{1}{2}, \frac{\alpha}{2}\right)}}\right)
$$

where $\hat{\sigma}$ is mle of $\sigma$ obtained from (3) and $G\left(n\left(\frac{\beta+1}{2}\right), \frac{1}{2}, q\right)$ is the $q$-th quantile of $\operatorname{Gamma}\left(n\left(\frac{\beta+1}{2}\right), \frac{1}{2}\right)$.

When $n$ is large, using (9), the approximated CI's at level $(1-\alpha)$ for $\mu$ and $\sigma$ are

$$
\left(\hat{\mu}-z_{1-\frac{\alpha}{2}} \sqrt{\frac{\beta-1}{n(2 \beta-1)}} \hat{\sigma}, \hat{\mu}+z_{1-\frac{\alpha}{2}} \sqrt{\frac{\beta-1}{n(2 \beta-1)}} \hat{\sigma}\right)
$$


and

$$
\left(\frac{\sqrt{2 n(\beta+1)} \hat{\sigma}}{\sqrt{2 n(\beta+1)}+z_{1-\frac{\alpha}{2}}}, \frac{\sqrt{2 n(\beta+1)} \hat{\sigma}}{\sqrt{2 n(\beta+1)}-z_{1-\frac{\alpha}{2}}}\right),
$$

respectively, where $z_{q}$ is the $q$-th quantile of standard normal distribution. When $\beta$ is unknown, the asymptotic confidence intervals can be obtained from (8). However, in practice, the sample size is usually small and distribution of the estimators are complex. In the following section we introduce the numerical method to obtain the CI for parameters.

\section{Confidence Interval of Parameters Using the Numerical Method}

When $\sigma$ and $\beta$ are known, we can use the following pivotal quantity

$$
\begin{aligned}
\Lambda & =\frac{\sqrt{n}(\bar{X}-\mu)}{\sigma} \\
& =\sqrt{n} \cdot \frac{\sum\left(\frac{X_{i}-\mu}{\sigma}\right)}{n}=\sqrt{n} \bar{Z},
\end{aligned}
$$

where $\bar{Z}=\frac{\sum_{i=1}^{n} Z_{i}}{n}$ and $Z_{i}=\frac{X_{i}-\mu}{\sigma} \sim S D N(\beta)$. The distribution of this pivotal quantity is complicated but independent of parameter $\mu$. One CI for $\mu$ based on $\Lambda$ is given by

$$
\left(\bar{X}+\Lambda_{\left(n, \frac{\alpha}{2}, \beta\right)} \frac{\sigma}{\sqrt{n}}, \bar{X}+\Lambda_{\left(n, 1-\frac{\alpha}{2}, \beta\right)} \frac{\sigma}{\sqrt{n}}\right)
$$

where $\Lambda_{(n, q, \beta)}$ is the $q$-th quantile of $\Lambda$ based on random sample of size $n>1$. This quantile can be obtained by the numerical method will be introduced in Section 6.

When $\sigma$ is unknown, we introduce the new pivotal quantity based on the sample mean and standard deviation as

$$
\Lambda^{\prime}=\frac{\bar{X}-\mu}{\frac{S}{\sqrt{n}}}=\frac{\bar{Z}}{\sqrt{\frac{\sum\left(Z_{i}-\bar{Z}\right)^{2}}{n(n-1)}}} .
$$

Note that $\frac{S^{2}}{\beta+1}$ is an unbiased estimator of $\sigma^{2}$. The distribution of this pivotal value is also complicated but independent of $\mu$ and $\sigma$ parameters. The $q$-th 
quantile of $\Lambda^{\prime}, \Lambda_{(n, q, \beta)}^{\prime}$, based on random sample of size $n>1$ is obtained by numerical method given below.

However, in this case one CI for $\mu$ is given by

$$
\left(\bar{X}+\Lambda_{\left(n, \frac{\alpha}{2}, \beta\right)}^{\prime} \frac{S}{\sqrt{n}}, \bar{X}+\Lambda_{\left(n, 1-\frac{\alpha}{2}, \beta\right)}^{\prime} \frac{S}{\sqrt{n}}\right) .
$$

We can also introduce the following pivotal quantity to obtain CI for $\sigma$

$$
Q=\frac{(n-1) S^{2}}{\sigma^{2}}=\sum\left(\left(\frac{X_{i}-\mu}{\sigma}\right)-\left(\frac{\bar{X}-\mu}{\sigma}\right)\right)^{2}=\sum\left(Z_{i}-\bar{Z}\right)^{2} .
$$

The distribution of $Q$ is also complicated but independent of $\mu$ and $\sigma$ parameters. Based on $Q$ the following CI for $\sigma$ is introduced

$$
\left(\frac{\sqrt{n-1} S}{\sqrt{Q_{\left(n, 1-\frac{\alpha}{2}, \beta\right)}}}, \frac{\sqrt{n-1} S}{\sqrt{Q_{\left(n, \frac{\alpha}{2}, \beta\right)}}}\right)
$$

where $Q_{(n, q, \beta)}$ denotes the $q$-th quantile of $Q$ which is obtained by the numerical method.

When $\beta$ is unknown we have not yet obtained pivotal quantities to provide such confidence intervals. In practice, $\mu$ and $\sigma$ are more interested. Therefore, we propose to replace $\beta$ with its mle in the CI corresponding to $\mu$ and $\sigma$ and given in (16) and (17), respectively.

\section{A Numerical Method to Calculate $\Lambda, \Lambda^{\prime}$ and $Q$}

Based on written program introduced in Section 2, a numerical method has been carried out to find the quantiles of the distribution of $\Lambda, \Lambda^{\prime}$ and $Q$ which are introduced in Section 5. For given $n$, we use the following algorithm:

Step 1: Draw a sample of size $n$ from $Z \sim S D N(\beta)$.

Step 2: Calculate $\Lambda, \Lambda^{\prime}$ and $Q$.

Both steps are repeated $\mathrm{K}$ times, then the $\frac{\alpha}{2}$-th and $\left(1-\frac{\alpha}{2}\right)$-th quantiles for $\Lambda, \Lambda^{\prime}$ and $Q$ are drived and used for the CI's introduced in (14), (16) and $(17)$.

A program in SPLUS-2000 is written by author to generate a sample from $D N(\mu, \sigma, \beta)$. As a numerical example, we draw a sample of size $n=50$ from $D N(\mu, \sigma, \beta)$, where $\mu=40, \sigma=5$ and $\beta=2$. The sample mean and variance are $\bar{x}=39.73$ and $S^{2}=80.56(S=8.98)$, respectively. 
The histogram of data is shown in Figure 2. The mle of parameters can be estimated from nlminb function in SPLUS-2000. The mle's are $\hat{\mu}=39.72$, $\hat{\sigma}=5.1$ and $\hat{\beta}=1.9$ (with start points $39.73,5.68,1.5$ and 11 iterations). Thus one CI for $\mu$ at level $95 \%$ based on $\beta=1.9$ is $\left(39.72-\frac{8.98}{\sqrt{50}} \times 2.04,39.72+\right.$ $\left.\frac{8.98}{\sqrt{50}} \times 1.98\right)=(37.13,40.23)$. Note that simulated quantiles by $K=10000$ repeats are $\Lambda_{(50,0.025,1.9)}^{\prime}=-2.04$ and $\Lambda_{(50,0.975,1.9)}^{\prime}=1.98$. Also at level $95 \%$ one simulated CI for $\sigma$ based on $Q$ with $\beta=1.9$ is given by $(4.64,5.85)$. Note that simulated quantiles by $K=10000$ repeats are $Q_{(50,0.025,1.9)}=183.5$ and $Q_{(50,0.075,1.9)}=115.4$.

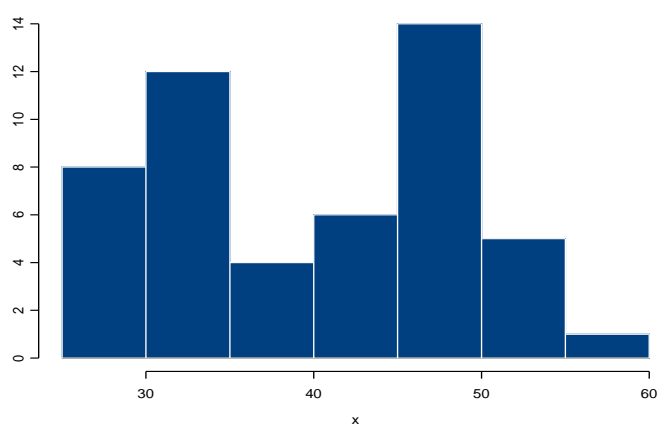

Figure 2. Histogram of the sample data

\section{Acknowledgement}

We would like to thank the referees and Dr. Daneshkhah for their helpful comments. This study was supported financing by Shahid Chamran University of Ahvaz.

\section{References}

Alavi, S.M.R. and Chinipardaz, R. (2011). A useful Distribution for Symmetric and Bimodal Data. Pioneer Journal of Theoretical and Applied Statistics, 1, 25-41. 
Alavi, S.M.R. and Chinipardaz, R. (2009). Form-Invariance Under Weighted Sampling. Statistics, 43, 81-90.

Fang, K.T., Kotz, S. and Ng, K.W. (1990). Symmetric Multivariate and Related Distributions. Chapman \& Hall, London.

Knight, K. (2000). Mathematical Statistics. Chapman and Hall, London.

Nadarajah, S. (2003). The Kotz type distribution with applications. Statistics, 37, 341-358.

Patil, G.P. (2002). "Weighted Distributions," In Encyclopedia of Environmetrics, 2369-2377, eds. Shaarawi, A. H. and Piegorsch, W. W., Chichester: John Wiley.

Patil, G.P. and Rao, C.R. (1977). "The weighted distributions: a survey of their applications," In Applications of Statistics, 383-405, ed. Krishnaiah, R. R., Amsterdam: North-Holland.

Stuart, A., Ord, J.K. and Arnold, S. (1999). Kendall's Advanced Theory of Statistics, 2A, 6ed., Oxford University press, London.

\section{Sayed Mohammad Reza Alavi}

Department of Statistics,

Shahid Chamran University,

Ahvaz, Iran.

email: alavi_m@scu.ac.ir 